\title{
A Hyper-Redundant Manipulator
}

\author{
"Hyper-redundant" manipulators have a very large number of actuatable \\ degrees of freedom. The benefits of hyper-redundant robots include \\ the ability to avaoid obstacles, increased robustness with respect to \\ mechanical failure, and the ability to perform new forms of robot lo- \\ comotion and grasping. The authors examine hyper-redundant ma- \\ nipulator design criteria and the physical implementation of one \\ particular design: a variable geometry truss.
}

\section{Introduction}

The word "redundant" is used in the context of robotic manipulators to indicate that the number of actuated degrees of freedom exceeds the minimal number required to perform a particular task. For instance, a manipulator required to position and orient an object in space needs six actuated degrees of freedom, and so a manipulator with seven or more is redundant with respect to this task. "Hyper-redundant" manipulators are redundant manipulators with a very large degree of redundancy. These manipulators can be analogous in morphology and operation to "snakes," "elephant trunks," or "tentacles." Because of their highly articulated structures, these robots are well suited for operation in highly constrained environments, and can be designed to have greater robustness with respect to mechanical failure than manipulators with a low degree of redundancy. Furthermore, the concept of hyper-redundancy can be generalized beyond manipulators to describe novel forms of robotic locomotion analogous to the motion of worms, slugs, and snakes.

Particular hyper-redundant designs have previously been referred to as: "highly articulated," "tentacle," "snake-like," "tensor-arm," "elephant trunk," "swan's neck," and "spine" (see [4] for specific references). To our knowledge, the earliest hyper-redundant robot designs/implementations date to the late 1960's [1]. Hirose and coworkers [12],[13] have implemented a large number of working high-dof systems. Numerous other authors have suggested hyper-redundant designs or developed hyper-redundant robot mechanisms. Examples include [16],[21],[24]. Many of these designs were driven to some extent by a particular application or operating environment/scenario. Figure 1 exemplifies the three major types of hyper-redundant manipulators: continuous, serial, and cascaded platforms.

The word 'hyper-redundant' was first used in [3] to capture the essence of these related concepts. Techniques for efficient and consistent kine-

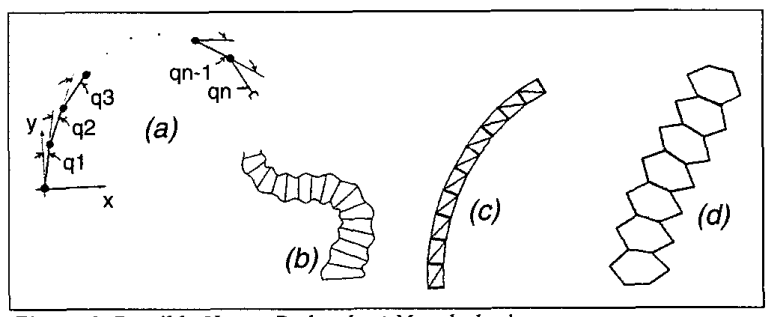

Figure 1. Possible Hyper-Redundant Morphologies.

matic analysis of hyper-redundant robots can be found in $[4],[5]$. These techniques have been used as the basis for novel obstacle avoidance, locomotion, and grasping schemes [3], [6], [2]. Furthermore, these techniques extend naturally to efficient models of hyper-redundant manipulator dynamics [7].

To date, hyper-redundant manipulators have remained largely a laboratory curiosity. There are a number of reasons for this: (1) standard kinematic techniques have not been particularly efficient or well suited to the needs of hyper-redundant robot task modeling; (2) the mechanical design and implementation of hyper-redundant robots has been perceived as unnecessarily complex; and (3) hyper-redundant robots are not anthropomorphic, and pose difficult programming problems. The authors have undertaken a broadly based investigation which we hope will eventually overcome many of the obstacles to practical hyper-redundant robot applications.

This paper presents the design and implementation of a thirty degree-of-freedom planar hyper-redundant manipulator. This manipulator was developed to demonstrate the feasibility of hyper-redundant manipulators for a variety of tasks. Experiments in hyper-redundant obstacle avoidance, grasping, object manipulation, dual arm grasping, and locomotion are presented.

Gregory S. Chirikjian, Dept. of Mechanical Engineering, Johns Hopkins University Joel W. Burdick, Dept. of Mechanical Engineering, Califormia Institute of Technology
The remainder of this paper is organized as follows: Section 2 reviews the concepts behind the authors' framework for hyper-redundant manipulator ki- 


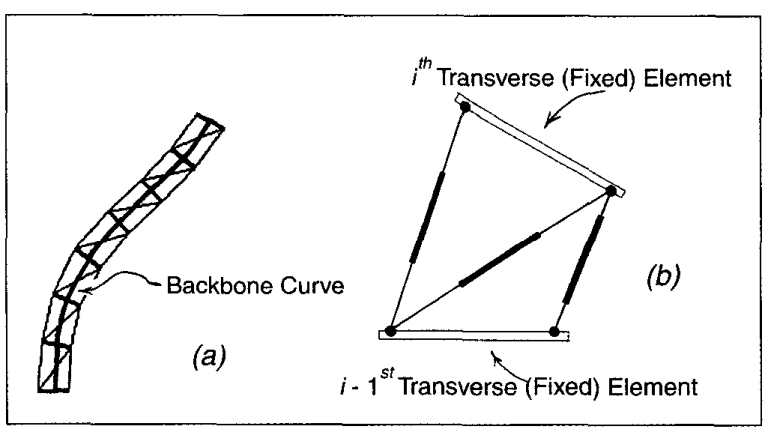

Figure 2. (a) Illustration of the 'Backbone Curve'; (b) a single module.

nematics. Section 3 overviews different hyper-redundant manipulator morphologies, includes some comments about the relative merits of each type, and describes the functional requirements for our system. Section 4 details the design and implementation of our hyper-redundant robot, and includes some philosophical arguments in favor of hyper-redundant robot systems for certain application domains. Section 5 reviews experiments performed with this system.

\section{A Continuum Approach to Kinematics}

The forward kinematics problem for serial chain manipulators is usually formulated using the Denavit Hartenberg framework. The inverse kinematics problem for redundant manipulators is usually solved using the Jacobian pseudo-inverse. That is, given a forward kinematic expression which specifies end-effector displacement $\vec{x}_{e e}$ in terms of the vector of joint displacements $\vec{q}_{e e}$ (or differential version of this expression):

$$
\vec{x}_{e e}=\vec{f}(q) \text { or } \dot{\vec{x}}_{e e}=\boldsymbol{J}(q) \dot{\vec{q}}
$$

an inverse kinematic solution of the form

$$
\dot{\vec{q}}=J^{+}\left(\dot{\vec{x}}_{e e}\right)+\left(1-J^{+} J\right) \dot{\vec{z}}
$$

is sought, where $\boldsymbol{J}^{+}=\boldsymbol{J}^{T}\left(\boldsymbol{J J}^{T}\right)^{-1}$. See [15] and references therein for discussion of this method.

While this method works well for appropriate choices of $\bar{z}$ when there are relatively few redundant degrees of freedom, more efficient alternatives exist for the hyper-redundant case.

We now review an alternate framework in which hyper-redundant robot kinematics is formulated in a way that we believe is more natural. Within this framework, "hyper-redundancy resolution" (the determination of joint angle trajectories which cause the manipulator to accomplish a specified task) is achieved using a backbone curve which captures the hyper-redundant robot's important macroscopic features, as shown in Figure 2(a). In effect, this method constrains a hyper-redundant manipulator to act as if it is 'glued' to a continuous backbone curve. That is, the backbone curve together with a fitting procedure serve as a continuum model for manipulators such as the n-link revolute manipulator or variable geometry truss (VGT) shown in Figures 1(a) and (c). Inverse kinematics and task planning operations are then reduced to the determination of the proper time varying behavior of the backbone curve. Backbone curve shapes which arise as the solution to inverse kinematic or other planning algorithms can be used to directly control the geometry of continuous morphology robots such as those constructed of bundles of pneumatic tubes or 'smart' structures made of shape memory alloy as depicted in Figure 1(b).

Continuum models are familiar in mechanics, where solids and fluids are represented as continuous media rather than a very large collection of particles. Because of the rather intuitive nature of this approach, it is not surprising that a number of authors have expressed similar ideas in the robotics literature [11], [18], [20], [24]. However, the real key to the success of this continuum approach is in determining the most physically meaningful way of parametrizing curves and restricting curve shapes to perform useful tasks. Backbone curves must be parametrized so as to capture important physical properties and limitations of real hyper-redundant robot structures. For example, a curve model must be flexible enough to account for the fact that hyper-redundant manipulators can be inextensible (fixed length), as in the n-link case with revolute joints, or extensible (variable length), such as a VGT. Furthermore, real hyper-redundant robots also have practical limits on bending, twisting, and extension/contraction which must be reflected in the backbone curve.

Extensible and inextensible planar and spatial curves can be parametrized in the general form:

$$
\vec{x}(s, t)=\int_{0}^{s} l(\sigma, t) \vec{u}(\sigma, t) d \sigma
$$

where $0 \leq s \leq 1$ is a parameter measuring position along the backbone curve. This parametrization can be viewed as follows: the curve is 'grown' from the base $(\vec{x}(0, t)=\overrightarrow{0})$ to end $(\vec{x}(1, t))$, where $\vec{u}(s, t)$ specifies the direction of growth and $1(\mathrm{~s}, \mathrm{t})$ specifies the amount of growth for each value of $s$ at time t. $\vec{x}(s, t)$ is a vector from the base of the manipulator to the point on the backbone curve at a given value of $s . \vec{u}(s, t)$ is the unit tangent vector to the curve at $s . l(s, t)$ controls the length of the curve tangent and assumes the general form:

$$
l(s, t)=1+\varepsilon(s, t)>0
$$

where $\varepsilon(s, t)$ is the local extensibility of the manipulator at point $s$ and time $t$. A positive value of $\varepsilon(s, t)$ indicates an extension, while a negative value corresponds to contraction of the curve at each value of $s$. Because $l(s, t)$ is a local property, some sections of an extensible manipulator may be extending while others are simultaneously contracting. For inextensible manipulators, or extensible ones which are artificially constrained to be inextensible, $\mathcal{\varepsilon}(s, t)=0 \forall s, t$.

Every hyper-redundant manipulator can be assigned a reference state (home position), which is achieved at some (possibly fictitious) time $t_{R}$. The reference state is chosen such that $\varepsilon\left(s, t_{R}\right)=0$. Thus, $s$ can be viewed as the normalized arclength of the manipulator backbone curve as measured in a reference 
state. If in this reference state, the manipulator is straight, $\vec{u}\left(s, t_{R}\right)=\vec{u}_{0}$ and $\vec{x}\left(s, t_{R}\right)=s \vec{u}_{0}$ for a constant unit vector $\vec{u}_{0}$ If the manipulator extends or contracts relative to this reference state, $s$ no longer represents arclength, but it still provides a means of parameterizing position.

Parametrizing curves in a physically meaningful way is only part of the problem of representing a hyper-redundant manipulator with a continuum model. We still must deal with the problem of algorithmically linking the behavior of the curve and the actual manipulator. For a variable geometry truss morphology (and other discretely segmented mechanisms), a "fitting" procedure is used to adapt the continuous curve solution to the discrete geometry. In the fitting procedure, the goal is to determine the individual actuator displacements which cause the manipulator to adhere as closely as possible to the continuous backbone curve. We restrict this discussion to the fitting procedure appropriate for the planar variable geometry truss, though fitting procedures for other geometries have been developed [4],[5].

Figure 2(b) shows one module of a planar variable geometry truss (VGT) manipulator. Assume that the robot consists of a serial chain of $n$ such modules. For this particular design the fitting procedure is extremely simple: (1) require that the centers of the base and top plates in the $t^{t h}$ module have the same positions as the points on the backbone curve at $s=(i-1) / n$ and $s=i / n$ respectively; (2) the base and top plates of each module must remain perpendicular to the curve. Therefore, the configuration of the $i^{\text {th }}$ module is completely specified by the segment of backbone curve: $(i-1) / n \leq s \leq i / n$. This means that given a backbone curve, the inverse kinematics of each module is computed separately because the kinematics of each segment does not influence the others. In the case of a VGT, the inverse kinematics is simply a matter of computing the actuated leg lengths as a function of the relative position and orientation of the top plate with respect to the bottom plate of a given module (which can be done with trigonometry). For a more formal analytical treatment of fitting procedures, see [5].

Given the above curve parametrization and a means of algorithmically linking the actual manipulator to the curve, the only remaining problem is that of restricting curve shapes so that the manipulator can perform useful tasks. Consider the planar case where a general unit tangent vector for the backbone curve can be written in the form

$$
\vec{u}(s, t)=[\sin \theta(s, t), \cos \theta(s, t)]^{T},
$$

where $\theta(s, t)$ is the angle that the unit tangent vector $\vec{u}(s, t)$ makes with the $y$ axis (measured clockwise). From the definition of the curvature of a planar curve, $\kappa(s, t)$, one finds that

$$
\theta(s, t)=\int_{0}^{s} \kappa(\sigma, t) l(\sigma, t) d \sigma,
$$

where curvature is taken to be positive for clockwise bending. This expression is useful because physical limitations on manipulator bending and extension/contraction can be enforced by ensuring that the backbone curve observes simple inequalities of the form: $|\kappa(s, t)|<\kappa_{\text {max }}$ and $|\varepsilon(s, t)|<\varepsilon_{\text {max }}$.
If the task which the manipulator is to perform is that of positioning an object in the plane, then it must have at least two degrees of freedom. On the other hand, if the goal is to perform such a simple task, there is no need to take advantage of all the manipulator's capabilities. Therefore, for end-effector placement tasks in the plane, hyper-redundancy resolution becomes a matter of restricting the behavior of the manipulator (via the curve model) to act as if it only has two degrees of freedom. In this way computations can be performed very efficiently. If the task changes, this algorithmic restriction will also change.

For the purpose of illustration with a closed form example, we will allow the curve to act as if it only has one bending and one extension 'mode.' One way to accomplish this is to choose functions of the form:

$$
\begin{aligned}
& \theta(s, t)=a_{1}(t) s \\
& l(s, t)=a_{2}(t) .
\end{aligned}
$$

The configurations resulting from this choice of modes are circular arcs (because the curvature is uniform, i.e., independent of $s$, and the length and curvature of these arcs are functions of $a_{1}(t)$ and $a_{2}(t)$. The forward kinematics for this class of hyper-redundant manipulator configurations is found by simply substituting (7) and (8) into (3) and (5), integrating, and evaluating at $s=1$ :

$$
\begin{aligned}
& x_{e e}(t)=x(1, t)=\frac{a_{2}[t]}{a_{1}[t]}\left(1-\cos a_{1}(t)\right) \\
& y_{e e}(t)=y(1, t)=\frac{a_{2}(t)}{a_{1}[t]} \sin a_{1}[t] .
\end{aligned}
$$

The inverse kinematics corresponding to the above choice of modes is:

$$
a_{1}(t)=2 A \tan 2\left(x_{e e}(t), y_{e e}(t)\right) ; \quad a_{2}(t)=\frac{a_{1}(t) y_{e e}(t)}{\sin a_{1}(t)}
$$

Thus, by restricting the curve to act as if it has 2 dof, the manipulator which is "glued" to the curve also acts as if it has 2 dof, and closed-form inverse kinematic solutions can be generated.

The generalization of this approach, in which $\theta(s, t)$ and $l(s, t)$ are each a weighted sum of base functions, is referred to as the modal approach to hyper-redundancy resolution [5]. An infinite variety of manipulator configurations can be generated corresponding to different choices of base functions for $\theta(s, t)$ and $l(s, t)$ when using this modal method. This method applies not only to end-effector placement, but also obstacle avoidance, grasping, and locomotion.

\section{Design Criteria}

In this section we examine the design criteria which determine the choice and arrangement of a hyper-redundant robot's actuators and mechanical structure. The selection of a particular morphology will obviously depend heavily upon the 
functional and operational requirements of a particular application. Some possible morphologies are represented in Figure 1. To obtain the maximum possible benefit from their highly articulated structures, hyper-redundant manipulators must not only be able to place their end-effectors at a specified location, but must also be able to do things that are difficult or impossible for mildly redundant and kinematically sufficient manipulators, such as:

- maneuver through highly constrained workspaces

- grasp objects by completely enveloping them

- generate peristaltic waves to manipulate objects.

These functional requirements arise in the following anticipated application scenarios:

- operations in highly constrained environments, such as: nuclear reactor steam generator maintenance; chemical sampling in buried toxic waste containers; and medical endoscopy.

- tentacle-like grasping and object reorientation such as: capturing and despinning free floating satellites; replacement of mechanical components in hard to reach areas of an automobile; and complex 'whole arm' manipulation experiments.

- exploration in complex environments such as: emergency response vehicles in burning or collapsed buildings; and sensor placement in complicated geological formations, such as lava tubes.

Naturally, no single design can be suitable for all applications. Rather than discuss the morphology-application relation, we will focus on the enumeration of broad design classes and their strengths and weaknesses. Section 4 reviews our actual implementation in the context of this discussion.

Hyper-redundant manipulators can be physically implemented using a variety of actuators and linkages, such as pneumatic bellows [22], [24], rubber gas actuator driven devices [10], a serial chain consisting of a large number of rigid links [[16]], [19], or a variable geometry truss [17], [18], [20], [23]. These morphologies can be roughly categorized into three main types: (1) discrete morphologies (Figure 1(a)) which have a large, but finite, number of rigid links; (2) continuous morphologies (Figure 1(b)) in which the robot actuation is distributed over the manipulator length and is continuously deformable; and (3) cascades of parallel platform modules such as variable geometry trusses (VGTs)(Figure 1(c)) or other hybrid parallel/serial mechanisms (Figure 1(d)). For the most part, the distinction in morphologies arises from the types of actuators chosen, and their means of deployment in the robot's structure.

In continuous morphologies, actuation is distributed through the robot structure, resulting in the ability to continuously deform the robot's local geometry. Continuous morphologies include robots based on flexible pneumatic and hydraulic actuators and actuator bundles. At first glance contin- uous manipulators would appear to be more maneuverable and highly articulated. However, their actual implementation can be problematical. Truly distributed actuation (akin to muscles in a tentacle or tongue [14]) is difficult to engineer in practice. Further, pneumatic and hydraulic schemes are not very suitable for locomoting hyper-redundant robots, as it is difficult to carry the pumps, regulators, and other required components in a self-propelled package.

Discrete morphologies are comprised of a finite number of non-distributed actuators. Examples of such systems include serial chain rigid link robots (with revolute and/or prismatic joints). Serial chain rigid link systems are an extension of traditional manipulator design methodology, have a simple kinematic structure, and have simple 'fitting' algorithms [4]. However, this morphology has inherently poor mechanical advantage, making it difficult to build one which can support its own weight when fully extended. In addition, serial chain designs are not very robust with respect to actuator failure.

Variable geometry truss systems have many advantages for practical hyper-redundant manipulator implementation. VGTs were originally studied in the context of large spacestructures, such as precision segmented mirrors. Examples in the literature are numerous, e.g., [17] [23]. More recently, variable geometry truss manipulators (VGTMs) have been proposed and investigated [18], [20]. Traditional VGTs and VGTMs can be differentiated by their actuation requirements. For large space structures, the primary role of the actuators and control system is to control structural resonances. Thus, these systems have actuators with high bandwidth and very little stroke. Conversely, VGTs for robotics applications require actuators with substantial stroke to effect significant changes in the structure geometry. We have chosen a VGT geometry for our system because of its inherent mechanical advantage, which is required for several of the experiments in hyper-redundant grasping. Section 4 will elaborate on this design, and other relative merits of VGT systems.

Most actuator technologies and implementations fall clearly into one category or the other. However, some actuators, such as tendons or shape memory alloys, can be deployed in either a continuous or discrete fashion. For example, tendons can be distributed throughout a hyper-redundant structure to provide continuous or discrete changes in geometry, depending upon the way they are attached to the robot structure. Tendons can be active, e.g. constructed from contracting shape memory alloys or contracting polymers. Alternatively, they can be passive, in which case they are actuated by pulling (typically with a d.c. servo motor). A number of tendon-based structures have been suggested and constructed [1]. Tendon based structures can be very light in weight. However, active tendons based on SMAs are typically slow in response. Passive tendons require remotely mounted actuators, and tendon routing becomes very complicated and impractical for the large numbers of tendons required to implement versatile spatial hyper-redundant robots. Further, nearly all tendon based designs implemented to date do not have complete kinematic functionality. That is, these designs can not provide twisting motion about the manipulator's 'backbone', nor do they have a general capability to implement broad changes in the torsion and curvature of the backbone. Consequently, they can only assume a restricted class of shapes. 
In the following section, we outline the development of our system, which possesses all of the required characteristics stated in this section.

\section{Manipulator Description}

For simplicity's sake, we have chosen to focus on the development of a planar hyper-redundant robot, leaving the development of a spatial system for future work. The primary functions of this robot are to demonstrate and validate our previous analytical work, and to serve as the basis for future work in hyper-redundant robot analysis, design, programming, and sensor-based operation. Consequently, this robot satisfies the functional requirements outlined in Section 3.

Our final design is a 30 degree-of-freedom VGT structure consisting of 10 identical three degree-of-freedom truss modules, or 'bays.' Each truss module (Figure 2) contains 3 prismatic joints. In effect, each bay is a planar parallel manipulator. The prismatic joints are actuated with D.C. servo motors and lead screw drives. The actuators can vary in length from a minimum of 12 inches to a maximum of 18 inches, can generate a force of 75 pounds during motion, and can withstand 225 pounds staticly. Each actuator is equipped with a linear potentiometer to measure its absolute displacement. The accuracy of the feedback potentiometer, and the backlash in the lead screw are both approximately 1 percent of maximum extension.

For manipulation and grasping experiments, casters are attached to the fixed (transverse) elements of the modules in order to allow low friction motion over the laboratory floor, which is the plane of manipulation. Detachable foam 'fingers' are used as the contact points for planar grasping operations. Because of its modular design, the robot can be easily separated into two 15 degree-of-freedom manipulators for dual arm manipulation experiments.

Figure 2(b) shows one module, or bay, of the truss robot. Let the length of a module (from fixed element to fixed element as measured in a reference configuration) divided by the width of the module (distance between actuator pivot points) be called the module aspect ratio. The fixed elements of the truss modules are designed so that each module's aspect ratio can be varied for different applications. A very high module aspect ratio leads to greater dexterity, but has reduced mechanical advantage. High aspect ratios are advantageous for obstacle avoidance in highly constrained spaces. Conversely, low aspect ratios provide greater mechanical strength at the expense of dexterity. For most applications, the module aspect ratios are uniformly sized, though they can be made non-uniform when necessary. For example, in manipulation, the modules closest to the base are subjected to greater forces and moments. Their aspect ratios can be reduced to improve their strength.

The robot system is controlled by a multiprocessing computer, which consists of Heurikon 68030 processing boards in a VME bus, which is in turn connected to a Sun Microsystems 4/260 computer. The WindRiver Systems VxWorks software system provides operating system and system management functions. The 68030 processors compute the kinematic, path planning, sensor processing, and task level coordination functions. Low level control of the actuator positions is also performed by 68030 processors. The Sun computer is used pri- marily for user interface and data storage.

As demonstrated by our design, hyper-redundant robots do have some inherent design and operating advantages.

First, hyper-redundant robot design is often amenable to simple modular implementation. In the planar case, each module consists of a planar parallel manipulator. For spatial manipulators, the module geometry can be a Stewart platform. Simple mechanical adjustments can alter the mechanical advantage properties of the modules to suit different needs.

Second, in most operational scenarios, only a small fraction of the robot actuators need to be used, minimizing their duty cycle and prolonging their life. Even so, such systems are highly fault tolerant with respect to numerous actuator failures. For instance, in this planar thirty degree of freedom manipulator, ninety percent of the actuators can fail, and the system will retain the ability to position and orient objects at the end-effector (though obstacle avoidance capability and the workspace volume would be severely reduced).

Third, assembly and maintenance of this hyper-redundant manipulator is extremely simple. In particular, the entire mechanism can be assembled from its constituent parts in less than one half hour (with 3 people working in parallel). Partial disassembly to replace actuators is trivial. The pins which fix the relative position of each actuator in the overall structure can be removed, and an actuator can be replaced in less than 5 minutes. For dual-arm experiments, the 10-bay truss can be 'split' into two 5-bay units (which are still hyper-redundant) in a matter of minutes also.

Such systems can also be weight efficient. This 30 degreeof-freedom robot can contract to a minimum length of 12 feet and extend to a maximum length of 18 feet. Yet the robot's mass is less than $55 \mathrm{~kg}$.

Of course, hyper-redundant robots have obvious disadvantages, and are best suited to niche applications. Small errors in each actuator position can accumulate into large base-totip position errors. Thus, hyper-redundant manipulators are at a disadvantage for precise positioning tasks, unless they use bracing, or are instrumented with numerous sensors and equipped with sensor-based control strategies which adjust for local errors. Another drawback is that the cost required to construct and control a system composed of a large number of actuators can be rather high. The problems of error rejection [9] and the development of new paradigms for producing inexpensive highly actuated robots [8] are the subject of current research.

\section{Experiments}

This section describes the task scenarios which have been evaluated with the 30 degree-of-freedom variable geometry truss described in the previous section. For some tasks the manipulator was used as a single unit, while in others the manipulator was split and the two halves were used separately.

Obstacle Avoidance: The theory developed in [3], [5] for obstacle avoidance is implemented in Figures 3-4. Here the manipulator is demonstrating a task which would be insurmountable by standard kinematically sufficient manipulators.

Single-Arm Grasping: The photos in Figure 5 show how a hyper-redundant manipulator can be used as a grasping device. In this figure, a single hyper-redundant manipulator is 


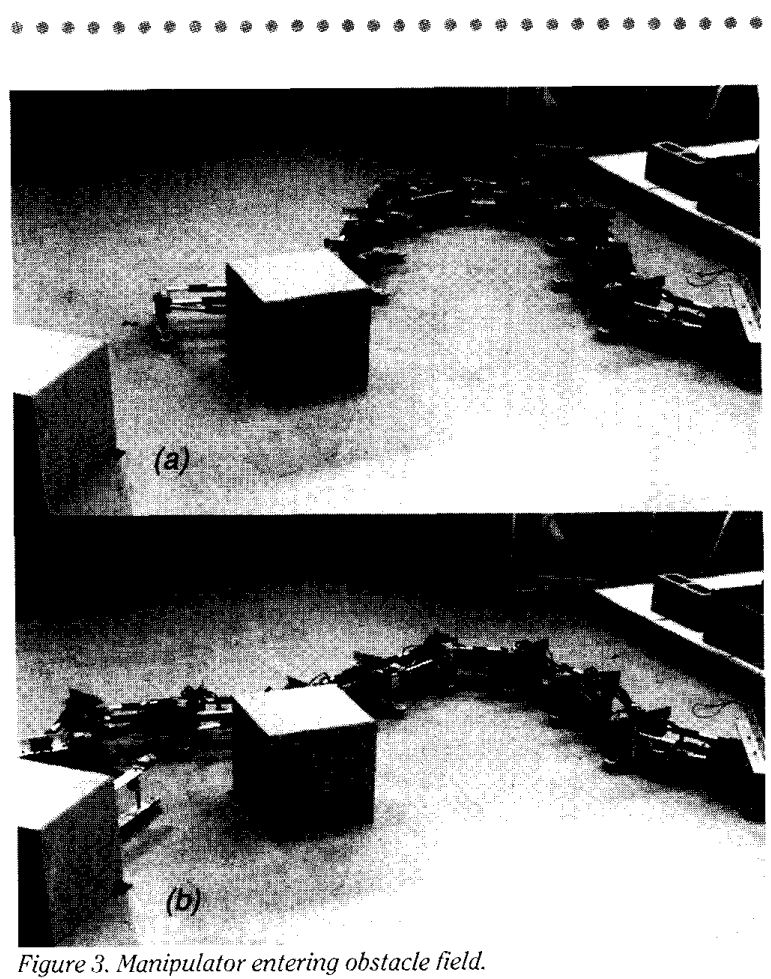

grabbing a mock Cold War era satellite, to be recovered from orbit.

Dual Arm Manipulation: A variant on the single arm grasping and manipulation scheme is shown in Figure 6. In this case, two hyper-redundant arms work together, as formulated in [4].

Locomotion: Figure 7 shows one half of the manipulator being used as a locomotion device. In particular, it is 'inch-

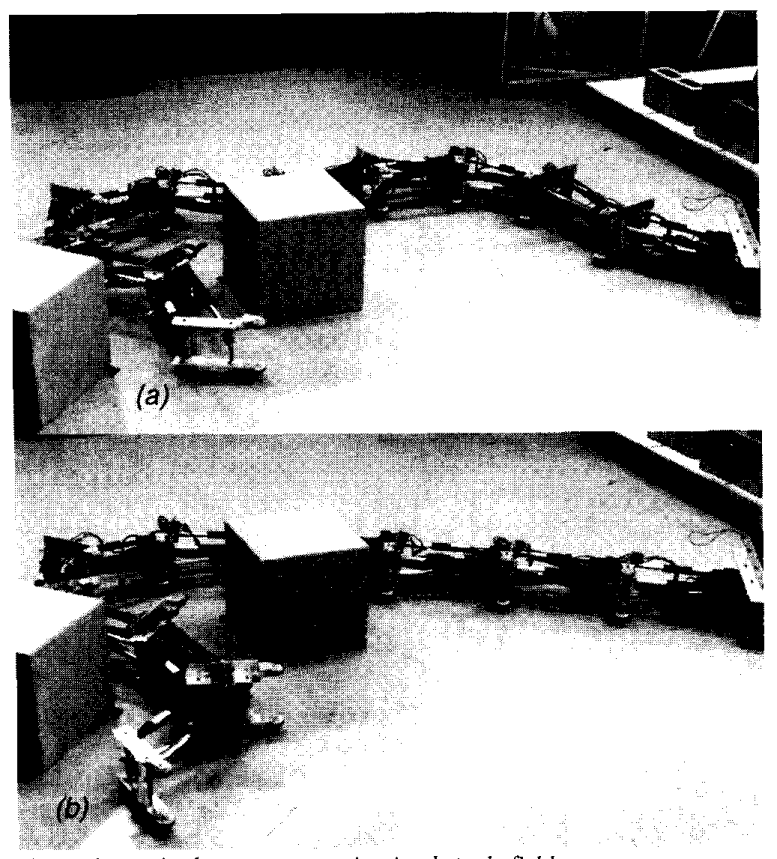

Figure 4. Manipulator maneuvering in obstacle field.

December 1994 worming' by expanding and contracting cyclically. In this scenario, the manipulator is turned on its side, and ratcheting wheels only allow unidirectional motion.

\section{Summary}

This paper presented the design and implementation of a 30 degree-of-freedom planar hyper-redundant robot. This system was developed to demonstrate and validate the authors' previous analytical investigations of hyper-redundant robot kinematics and redundancy resolution. In previous work, we developed techniques which are efficient and sufficiently general to enable real-time inverse kinematics and motion planning for this complex class of robots. Further, this basic framework has been used to develop novel obstacle avoidance, locomotion, and grasping algorithms. The experiments detailed in this paper demonstrate the validity of our previous work. Further, our experience in constructing this system shows that hyperredundant manipulator designs do not need to be complex. Our hope is that these analytical and experimental investigations will bring the concept of hyper-redundancy closer to use in the field.

\section{Acknowledgments}

This work was sponsored in part by a NASA Graduate Student Researchers Program fellowship (while the first author was a student), the Caltech President's Fund, \# PF-331, and the National Science Foundation, grant MSS-901779.

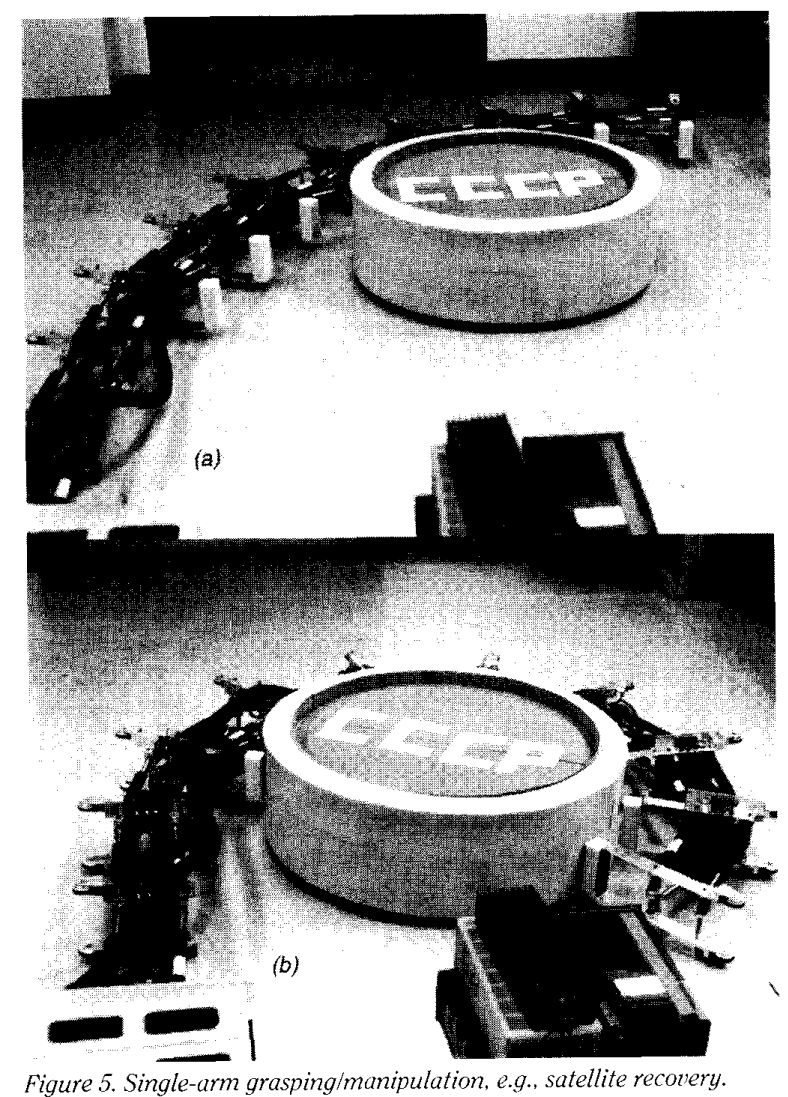

IEEE Robotics \& Automation Magazine . 


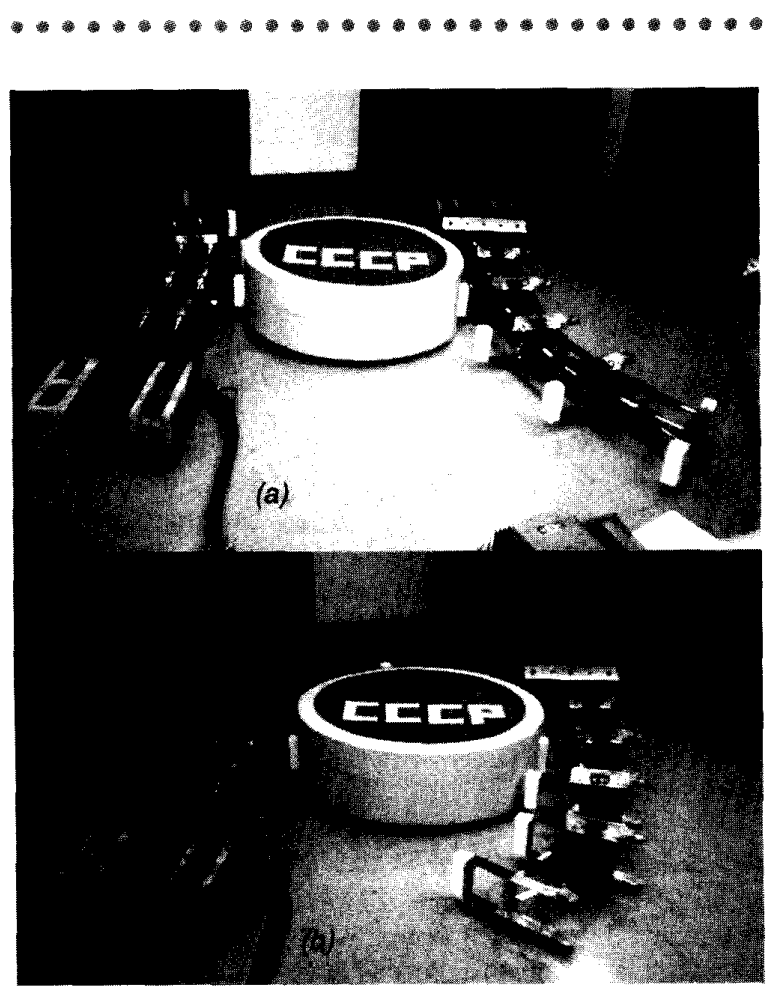

Figure 6. Dual-arm grasping/manipulation, e.g., satellite recovery.

\section{References}

[1] Anderson, V.V., Horn, R.C., "Tensor-arm Manipulator design," ASME Trans., Vol. 67-DE-57, pp. 1-12, 1967.

[2] Burdick, J.W., Radford, J., Chirikjian, G.S., "A Sidewinding Locomotion Gait for Hyper-Redundant Robots," Journal of Advanced Robotics (in press)

[3] Chirikjian, G.S., Burdick, J.W., "An Obstacle Avoidance Algorithm for Hyper-Redundant Manipulators," Proc. 1990 IEEE International Conference on Robotics and Automation, Cincinnati, $\mathrm{OH}$, May 13$18,1990$.

[4] Chirikjian, G.S., "Theory and Applications of Hyper-Redundant Robotic Manipulators," Ph.D Dissertation, Department of Applied Mechanics, Division of Engineering and Applied Science, California Institute of Technology, June 1992.

[5] Chirikjian, G.S., Burdick, J.W., "A Modal Approach to Hyper-Redundant Manipulator Kinematics," IEEE Transactions on Robotics and Automation, June, 1994.

[6] Chirikjian, G.S., Burdick, J.W., "The Kinematics of Hyper-Redundant Robot Locomotion," IEEE Transactions on Robotics and Automation (in press)

[7] Chirikjian, G.S., "Hyper-Redundant Manipulator Dynamics: A Continuum Approximation," Journal of Advanced Robotics (in press)

[8] Chirikjian, G.S.,"A Binary Paradigm for Robotic Manipulators," 1994 IEEE International Conference on Robotics and Automation, San Diego, CA, May 1994.

[9] Choset, H., Burdick, J., "Sensor Based Planning and Nonsmooth Analysis," 1994 IEEE International Conference on Robotics and Automation, San Diego, CA, May 1994.

[10] Fukuda, T., Hosokai, H., Uemura, M., "Rubber Gas Actuator Driven by Hydrogen Storage Alloy for In-pipe Inspection Mobile Robot with Flexible Structure," Proceedings 1989 IEEE International Conference on Robotics and Automation, Scottsdale, Arizona, pp. 18471852, May 1989.

[11] Hayashi, A., Kuipers, B., "A Continuous Approach to Robot Motion Planning with Many Degrees of Freedom," Proceedings, IROS'92,

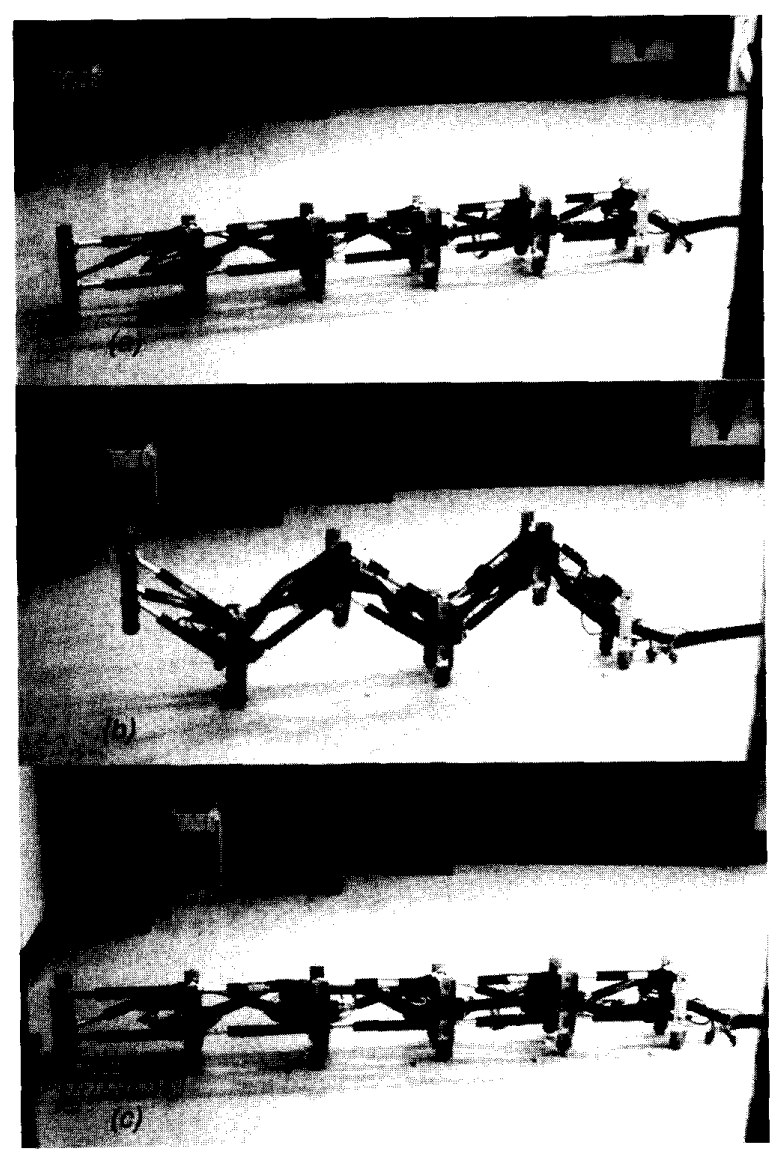

Figure 7. Inchworm locomotion.

pp. 1935-1942, Raleigh, NC, July 7-10, 1992.

[12] Hirose, S., Umetani, Y., "Kinematic Control of Active Cord Mechanism With Tactile Sensors," Proceedings of Second International CISM-IFT Symposium on Theory and Practice of Robots and Manipulators, pp. 241-252, 1976.

[13] Hirose, S., Yokoshima, K., Ma, S., "2 DOF Moray Drive for Hyper Redundant Manipulator," Proceedings, IROS'92, pp. 1735-1740B, Raleigh, NC, July 7-10, 1992.

[14] Kier, W.M., Smith, K.K., "Tongues, tentacles and trunks: the biomechanics of movement in the muscular-hydroststs," Zoological Journal of the Linnean Society, Vol. 83, pp. 307-324, 1985

[15] Klein, C.A., Huang, C.H., "Review of the Pseudoinverse for Control of Kinematically Redundant Manipulators," IEEE Transactions on Systems, Man and Cybernetics, Vol. SMC-13, No. 2, pp. 245-250, March 1983.

[16] Kobayashi, H., Shimemura, E., Suzuki, K., "A Distributed Control for Hyper Redundant Manipulator," Proceedings, IROS'92, pp. 1958 1963, Raleigh, NC, July 7-10, 1992.

[17] Miura, K., Furuya, H., "Variable Geometry Truss and Its Application to Deployable Truss and Space Crane Arm," Acta Astronautica, Vol. 12, No. 7/8, 1985, pp. 599-607.

[18] Naccarato, F., Hughes, P.C., "Inverse Kinematics of Variable-Geometry Truss Manipulators," Journal of Robotic Systems Vol. 8, No. 2, pp. 249-266, 1991.

[19] Resnik, D., Lumelsky, V., "Motion Planning with Uncertainty for Highly Redundant Kinematic Structures I. 'Free Snake' Motion," Proceedings, IROS'92, pp. 1747-1752, Raleigh, NC, July 7-10, 1992.

[20] Salerno, R.J., Reinholtz, C.F., Robertshaw, H.H., "Shape Control of High Degree-of-Freedom Variable Geometry Trusses," Proceedings 
of the Workshop on Computational Aspects in the Control of Flexible Systems, Part 2, Williamsburg, VA, July 12-14, 1988.

[21] Shahinpoor, M., Kalhor, H., Jamshidi, M., "On Magnetically Activated Robotic Tensor Arms," Proceedings of the International Symposium on Robot Manipulators: Modeling, Control, and Education, Nov. 12-14, 1986, Albuquerque, New Mexico.

[22] Suzumori, K., Iikura, S., Tanaka, H., "Development of Flexible Microactuator and Its Applications to Robotic Mechanisms," 1991 IEEE Conference on Robotics and Automation, Sacramento, CA April, 1991.

[23] Wada, B., "Adaptive Structures: An Overview," J. Spacecraft, Vol.27. No. 3., May-June 1990

[24] Wilson, J.F., Mahajan, U., "The Mechanics and Positioning of Highly Flexible Manipulator Limbs," Journal of Mechanisms, Transmissions, and Automation in Design. Vol, 111, June 1989.

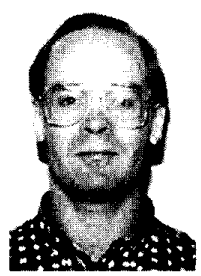

Joel W. Burdick is an associate professor in the Dept. of Mechanical engineering at the California Institute of Technology. He received his B.S. from Duke University and his Ph.D. from Stanford University. He is a recipient of the NSF Presidential Young Investigator Award, the Office of Naval Research Young Investigator Award, and is a FeynmanHughes Fellow. His current interests are in the areas of robotic locomotion, hyper-redundant robotics, sensor based motion planning, and medical applications of robotics.

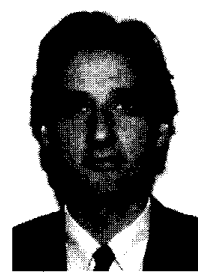

Gregory S. Chirikjian received the B.S.E. degree in Engineering Mechanics and the M.S.E. degree in Mechanical Engineering in 1988 while also fulfilling requirements for a B.A. degree in Mathematics, all from The Johns Hopkins University. Between 1988 and 1992 he was a graduate student at the California Institute of Technology, where he received the Ph.D. degree in Applied Mechanics. Since the summer of 1992 he has been an Assistant Professor in the Department of Mechanical Engineering at Johns Hopkins, and started the robotics program there. His general interests are in the kinematic analysis, design, and implementation of 'hyper-redundant,' 'metamorphic,' and 'binary manipulators.' He received an NSF National Young Investigator award in 1993 and a Presidential Faculty Fellows Award in 1994 to continue work in these areas.

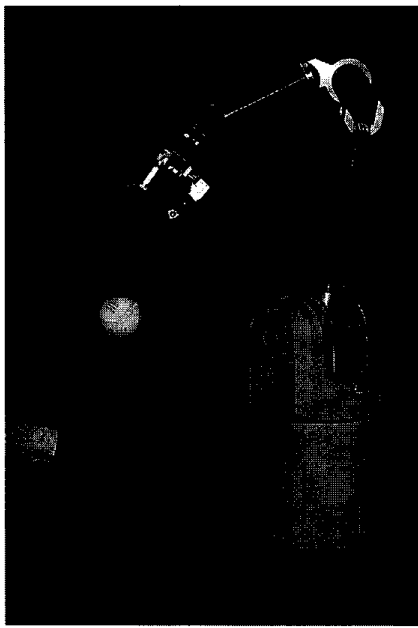

For more information contact William Townsend

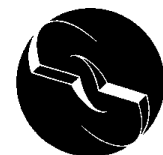

INNOVATIVE
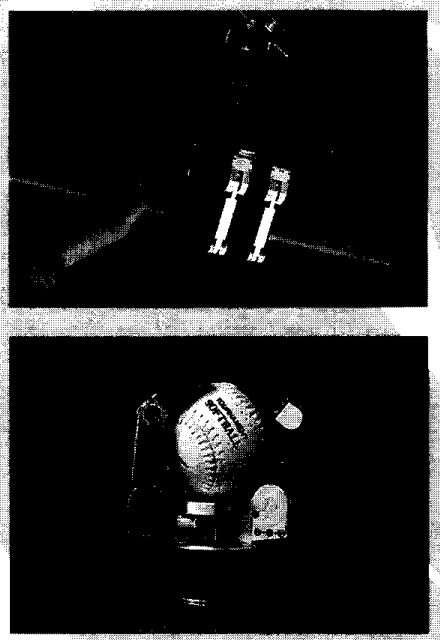

LI G H T WEIGH T

ECONOMICA L

Barrett Technology Inc. 545 Concord Avenue Cambridge, MA 02138 USA Tel 617868-7716 Fax 617 868-7737 